\title{
Hydrocephalus associated with childhood nonaccidental head trauma
}

\author{
*Sudhakar Vadivelu, D0,1 Harold L. Rekate, MD, ${ }^{1}$ Debra Esernio-Jenssen, MD, ${ }^{2}$ \\ Mark A. Mittler, MD, ${ }^{1}$ and Steven J. Schneider, MD'
}

\begin{abstract}
${ }^{1}$ The Cushing Neuroscience Institute and Department of Neurosurgery, Hofstra Northwell School of Medicine at Cohen Children's Medical Center and Northwell Health System, Manhasset, New York; and 'Department of Pediatrics, University of Florida School of Medicine at Shands Children's Hospital, Gainesville, Florida
\end{abstract}

\begin{abstract}
OBJECTIVE The incidence of posttraumatic ventriculomegaly (PTV) and shunt-dependent hydrocephalus after nonaccidental head trauma (NAHT) is unknown. In the present study, the authors assessed the timing of PTV development, the relationship between PTV and decompressive craniectomy (DC), and whether PTV necessitated placement of a permanent shunt. Also, NAHT/PTV cases were categorized into a temporal profile of delay in admission and evaluated for association with outcomes at discharge.

METHODS The authors retrospectively reviewed the cases of patients diagnosed with NAHT throughout a 10-year period. Cases in which sequential CT scans had been obtained $(n=28)$ were evaluated for Evans' index to determine the earliest time ventricular dilation was observed. Discharge outcomes were assessed using the King's Outcome Scale for Childhood Head Injury score.
\end{abstract}

RESULTS Thirty-nine percent (11 of 28) of the patients developed PTV. A low admission Glasgow Coma Scale (GCS) score predicted early PTV presentation (within < 3 days) versus a high GCS score (> 1 week). A majority of PTV/NAHT patients presented with a subdural hematoma (both convexity and interhemispheric) and ischemic stroke, but subarachnoid hemorrhage was significantly associated with PTV/NAHT $(p=0.011)$. Of 6 patients undergoing a DC for intractable intracranial pressure, $4(67 \%)$ developed PTV $(p=0.0366)$. These patients tended to present with lower GCS scores and develop ventriculomegaly early. Only 2 patients developed hydrocephalus requiring shunt placement.

CONCLUSIONS PTV presents early after NAHT, particularly after a DC has been performed. However, the authors found that only a few PTVINAHT patients developed shunt-dependent hydrocephalus.

http://thejns.org/doi/abs/10.3171/2016.8.FOCUS16266

KEY WORDS biomarker; child abuse; excitotoxicity; hypoxia-ischemia; shaken baby syndrome; traumatic brain injury

$\mathrm{T}$ HE natural history of hydrocephalus after nonaccidental head trauma (NAHT) has not been evaluated. This is partly due to the extremely dismal prognosis that NAHT portends and to the fact that it often occurs within 24 hours, correlating with a significant rate of mortality. As a matter of fact, the Center for Disease Control and Prevention in a Spring 2008 report (http:// www.cdc.gov/ViolencePrevention) listed NAHT as the leading cause of childhood death; the incidence reported in Pennsylvania, for example, was 14.7-26.0 cases per 100,000 person-years. ${ }^{11}$ This high incidence is not unique to the United States, as a similar rate was observed internationally: $12.8-21$ cases per 100,000 person-years in
England ${ }^{8}$ for instance, and $14.7-19.6$ cases per 100,000 person-years in New Zealand. ${ }^{10}$ Collectively, these statistics suggest that outcomes after NAHT are universally poor, with only $50 \%$ of patients admitted for NAHT living beyond the first 24 hours after injury and with reintegration into society difficult in $50 \%$ of surviving children due to severe morbidity. ${ }^{12}$ These poor outcomes may be in part due to the effects of hydrocephalus.

The incidence of ventricular dilation after traumatic head injury may be as high as $39 \%-44 \%$, and on average, this pathology becomes apparent at the 4-week postinjury interval. ${ }^{13,15}$ The incidence of posttraumatic ventriculomegaly (PTV) is unclear, as is that of subsequent development

ABBREVIATIONS CPCT = child protection consultation team; GCS = Glasgow Coma Scale; IVH = intraventricular hemorrhage; $\mathrm{KOSCHI} \mathrm{=} \mathrm{King's} \mathrm{Outcome} \mathrm{Scale} \mathrm{for}$ Childhood Head Injury; NAHT = nonaccidental head trauma; $\mathrm{PTV}=$ posttraumatic ventriculomegaly; $\mathrm{SAH}=$ subarachnoid hemorrhage; $\mathrm{SDH}=$ subdural hematoma; $\mathrm{TBI}=$ traumatic brain injury.

SUBMITTED June 28, 2016. ACCEPTED August 16, 2016.

INCLUDE WHEN CITING DOI: 10.3171/2016.8.FOCUS16266.

* Drs. Mittler and Schneider contributed equally to this work. 
to shunt-dependent hydrocephalus after NAHT. Marmarou et al. ${ }^{13}$ classically used a dye injection method to demonstrate altered CSF dynamics due to poor absorptive properties associated with subarachnoid hemorrhage (SAH) or intraventricular hemorrhage (IVH) in a majority of patients. Alternatively, operative procedures such as decompressive craniectomies have also been implicated in playing a role in promoting the development of hydrocephalus ${ }^{2,22}$ and subsequently could result in poorer outcomes. . $^{214,19}$

Here, we evaluated our cohort of patients with perpetrator-identified NAHT to address the involvement of hydrocephalus. We quantified the cumulative incidences of PTV and shunt-dependent hydrocephalus. We analyzed the data for epidemiological factors and radiographic signs associated with these primary findings. In addition, we questioned whether secondary factors such as a delay in seeking medical care and/or a decompressive craniectomy promoted hydrocephalus.

\section{Methods}

With approval from the institutional review board, we performed a retrospective, single-center, chart review of all patients who were evaluated by the medical child protection consultation team (CPCT) and who were reported to the New York State Central Register, documented between the 2001 and 2010. ${ }^{20}$ The total number of NAHT patients registered was 48. Inclusion criteria included cases in which the perpetrator was specifically identified by the Child Protection Services of Nassau, Suffolk, Brooklyn, and Queens counties and in which the perpetrator was considered the primary suspect in a criminal trial or was convicted by the local/state law enforcement agencies., ${ }^{4,20}$ Fourteen cases were excluded from this review because perpetrator status could not be identified, and 6 of the 34 cases in which a perpetrator was identified were excluded because the charts could not be reviewed at the time of this study as they were under review in preparation for a criminal trial or were being used during a criminal trial. The remaining 28 cases were evaluated by our hospital CPCT and pediatric neurosurgical department during the study period and included a multidisciplinary evaluation during admission.

The diagnosis of NAHT was indicated after clear evidence of intracranial injury was demonstrated on radiographs and after careful evaluation by CPCT. ${ }^{4,20}$

All admission CT scans and subsequent CT and MRI scans were collected and reviewed by both a neuroradiologist and neurosurgeon. PTV was defined using the Evans' index $(\geq 0.30)$. Hydrocephalus requiring shunt placement was determined by the Evans' ratio linear index for ventriculomegaly and persistent or worsening symptoms. ${ }^{15,16} \mathrm{We}$ determined the temporal profile of each case by identifying the time of injury/ictus as verified by the child protection and criminal investigation teams, regardless of whether the stated mechanism was accurate. We then identified the time of admission to the pediatric emergency room. This elapsed time was then categorized into the following temporal classification: 1 ) no delay, $0-6$ hours; 2 ) moderate delay, 6-12 hours; and 3) severe delay, > 12 hours. ${ }^{20}$

Outcomes assessments took into account the following variables: serial imaging, in-hospital morbidity, and neurosurgical interventions. Discharge outcomes were graded using the King's Outcome Scale for Childhood Head Injury $(\mathrm{KOSCHI})$ scores $(1=$ death, $2=$ vegetative state, 3 $=$ severe disability, $4=$ moderate disability, and $5=\operatorname{good}$ recovery). ${ }^{1,20}$

Statistical assessment involved the Biostatistics Unit of The Feinstein Institute for Medical Research and included univariate analysis of the patient age, sex, Glasgow Coma Scale (GCS; low score of 3-8; high score of 9-15), SAH, IVH, ischemic stroke, subdural hematoma (SDH), PTV, shunt-dependent hydrocephalus, decompressive craniectomy, admission delay, and KOSCHI score. ${ }^{20}$ For statistical purposes, scoring was represented in nonparametric ranks to preserve the order of the scoring. A Kruskal-Wallis test or Mann-Whitney U-test was performed for all univariate analyses, and a scatter plot was generated to represent statistical findings. Associations between categorical variables (admission delay, SDH, infarct, SAH, IVH, and midline shift) and PTV during hospitalization were examined using Fisher's exact test. Associations between continuous variables (age, GCS score at admission) and PTV during hospitalization were examined using exact logistic regression. All data were evaluated using SAS version 9.2 statistical software (SAS Institute, Inc.). A p value $<0.05$ was considered significant.

\section{Results}

\section{Incidence of Ventriculomegaly and Time of Presentation}

Of the 28 children diagnosed with NAHT, we identified $11(39 \%)$ with ventricular dilation according to the Evans' index (Table 1). Of NAHT patients presenting with low GCS scores $(\mathrm{n}=16), 7$ developed PTV $(44 \%)$, whereas of 12 NAHT patients presenting with high GCS scores, 4 developed PTV (33\%) (Table 1). Despite the fact that more NAHT patients presented with low GCS scores, GCS score on arrival did not prove predictive of subsequent hydrocephalus development. In our assessment of the earliest time of PTV presentation, PTV occurred as early as within 1 day of trauma but more commonly within 10 days of trauma (82\%; 9 of 11; Fig. 1 upper).

\section{Radiological Features Associated With PTV}

The most common radiological finding observed in our patients was SDHs; additionally, we observed a high incidence of retinal hemorrhages, a low presenting GCS score, higher male to female sex ratio, and age of less than 2 years, as previously described in our previous report. ${ }^{20}$ We investigated the association of additional radiological findings specifically to those NAHT patients who developed PTV ( $\mathrm{n}=11$; Table 1). Eight patients $(73 \%)$ had hemispheric SDHs and 6 patients $(55 \%)$ had interhemispheric or tentorial SDHs. Additionally, in 7 patients (64\%), the CT scan displayed hypodensities suggestive of ischemic stroke, another predominant sign. Despite these trends, only SAH, seen in 3 patients (27\%), proved significant ( $\mathrm{p}$ $=0.011)$. Historically, SAH and IVH are thought to contribute to ventricular dilation, but in only 1 case of PTV (1\%) was IVH observed, and this was not statistically significant (Table 1). 
TABLE 1. Summary of PTV data and their associated radiological findings

\begin{tabular}{lcc}
\hline \multicolumn{1}{c}{ Characteristic } & No. of Patients & p Value \\
\hline PTV incidence in NAHT & & \\
\hline Overall PTV incidence & $11 / 28(39 \%)$ & \\
\hline PTV incidence w/o craniectomy & $7 / 22(32 \%)$ & \\
\hline PTV incidence w/ craniectomy & $4 / 6(67 \%)$ & 0.0366 \\
\hline PTV \& GCS & & \\
\hline Mild/moderate NAHT (GCS Score 9-15) & $4 / 12(33 \%)$ & \\
\hline Severe NAHT (GCS Score 3-8) & $7 / 16(44 \%)$ & \\
\hline Permanent VPS placed & $2 / 28(7 \%)$ & \\
\hline VPS placed after craniectomy $\dagger$ & $1 / 6(17 \%)$ & \\
\hline Admission CT findings $\ddagger$ & & \\
\hline Convexity SDH (unilat and bilat) & 8 \\
\hline Interhemispheric/tentorial SDH & 6 \\
\hline SAH & 3 & 0.011 \\
\hline IVH & 1 & \\
\hline Ischemic stroke & 7 & \\
\hline
\end{tabular}

VPS $=$ ventriculoperitoneal shunt.

* Both cases presented with severe NAHT (GCS score of 3-8) and without delayed hospital admission.

† Limited temporal craniectomy with large superior frontoparietal craniotomy.

‡ Total number of PTV/NAHT cases was 11.

\section{Temporal Profile of Delay in Admission and PTV}

We recently reported on the concern about delays in obtaining acute medical care after NAHT, questioning the impact that delayed treatment may have on in-hospital discharge outcomes, including data on inpatient survival analysis. Patients who had a moderate delay in care-in other words, those who arrived to the hospital between 6 and 12 hours after onset of the first reported symptom (injury ictus) $)^{18,20,21}$ - had demonstrated worst outcomes compared with patients who presented without delay (0-6 hours postinjury ictus) or after a significant delay ( $\geq 12$ hours postinjury ictus). ${ }^{20}$ We questioned if time between injury and seeking medical care had any role in observations of PTV. Seven (64\%) of 11 patients with severe head injury (a low GCS score) had PTV, and this imaging finding was demonstrated within 3 days after injury. A majority of these patients presented without delay in arrival (0-6 hours) (Table 2). However, the other 4 patients (36\%), those with high a GCS score, had PTV, but it was demonstrated at least a week after injury. When we performed a subgroup analysis based on delay in arrival, we found that cases in which patients had a no or moderate delay in seeking medical care were associated with PTV development before 1 week postinjury, and in those cases in which patients arrived after severe delay ( $>12$ hours) PTV developed more than 2 weeks after injury (Fig. 1 lower). Low GCS score and no or moderate time delay in arrival for medical care were associated with earlier demonstration of PTV.

\section{Ventriculomegaly and Discharge Outcome}

We considered whether development of PTV has any impact on hospital discharge outcomes by comparing pa-
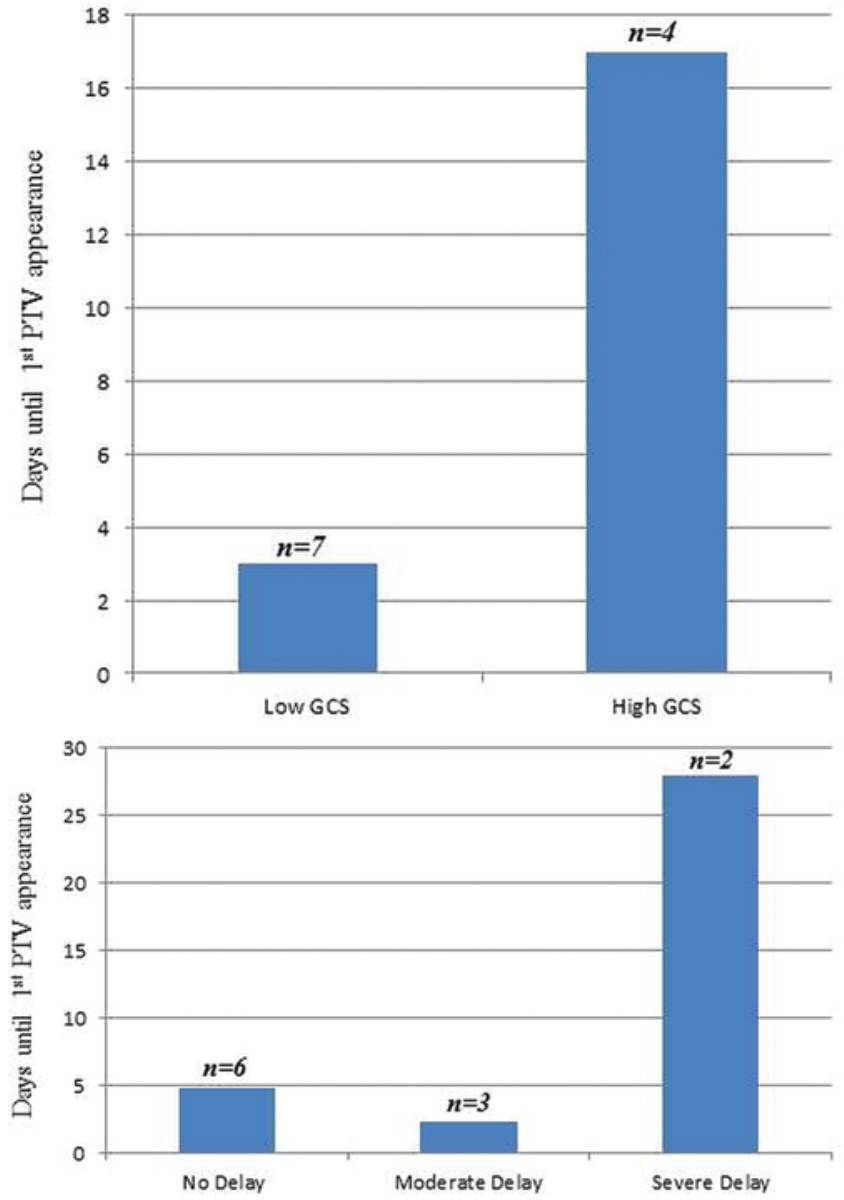

FIG. 1. Bar graphs showing the development of PTV after NAHT presentation in patients with a low or high GCS score (upper) and with or without delay in seeking acute medical care after injury (lower).

tients with and without PTV. Considering patients with early mortality who may not have undergone serial scanning, we were unable to perform any meaningful statistical comparison. However, when we examined those NAHT patients demonstrating PTV early $(<1$ week) versus late ( $>1$ week), poorer outcomes at inpatient discharge were observed in patients with early PTV than in those presenting with late PTV (KOSCHI score < 3; Fig. 2).

\section{PTV and Decompressive Craniectomy}

Decompressive craniectomies may be life-sustaining measures in patients with traumatic brain injury (TBI). Postoperative sequelae in such patients have been a subject of recent debate, including the development hydrocephalus. Here, we analyzed NAHT/PTV patients who underwent decompressive craniectomy. No patient had PTV on arrival at our hospital. Large frontotemporoparietal craniectomies $(n=5)$ or a temporal craniectomy $(n=1)$, which included dural openings, were performed in a total $6(21 \%)$ of 28 NAHT patients when significant cerebral edema or mass effect was present due to either intracranial findings on CT scans or intractable intracranial pressure. PTV was observed to occur in 4 of these patients (67\%). NAHT patients who did not undergo a decompressive craniectomy 
TABLE 2. Hospital admission GCS score and temporal profile of delay in arrival scores in NAHT/PTV patients

\begin{tabular}{cccc}
\hline & \multicolumn{3}{c}{ Delay in Seeking Care } \\
\cline { 2 - 4 } GCS Score & None & Moderate & Severe \\
\hline $3-8$ & 5 & 2 & 0 \\
\hline $9-15$ & 1 & 1 & 2 \\
\hline
\end{tabular}

were also observed to develop PTV (7 of 22 patients; $32 \%$ ) (Table 1). We asked whether those NAHT patients who underwent a decompressive craniectomy were more likely to be associated with PTV. We found decompressive craniectomy to be statistically significantly associated with NAHT/PTV development ( $\mathrm{p}=0.0366$; Table 1).

\section{Shunt Dependence}

Only 2 cases $(7 \%)$ of NAHT/PTV required permanent CSF shunting (Table 1). One of the patients had undergone a full frontotemporoparietal craniectomy. With only 2 cases, there was no statistical analysis that could address the role of decompressive craniectomy on shunt-treated hydrocephalus. Overall, shunted hydrocephalus was observed to occur infrequently in our cohort.

\section{Discussion}

To the best of our knowledge, this is the first study to examine the natural history of hydrocephalus after NAHT in children. Hydrocephalus may represent an important factor in discharge outcomes. Dias et al. documented that imaging findings such as intraparenchymal hypodensities on CT scans may be present as early as 3 hours after injury in contrast to historical documentation of presentation 6-24 hours after injury. Furthermore, chronic SDHs may present as early as 20 hours, much earlier than the 1-4 weeks traditionally viewed. ${ }^{3}$ For these reasons, we questioned whether the incidence of PTV and hydrocephalus in NAHT patients was similar to the incidence of PTV and hydrocephalus in other etiologies of TBI. To address these concerns, we first evaluated PTV, a radiological sign that can be demonstrated, with or without clinical correlation. We found the incidence of PTV in our series to be $38 \%$, on par with more general studies of TBI (adult TBI/PTV approximately 44\%). ${ }^{13}$ One important limitation of our study observations was nonprotocolled neuroimaging surveillance. We did not acquire routine CT scans and instead obtained imaging studies based on clinical parameters including neurological examination. In previous work, we questioned the post-NAHT time to seeking medical care as an important variable, ${ }^{20}$ and this, in addition to findings reported here, may prompt future investigation into the early need for serial imaging. The second limitation was that we used the Evans' index as a linear index common to several neuroradiology divisions across a large number of children's hospitals. Several studies including a recent report directly assessing the various methods have suggested the frontooccipital horn ratio may better serve as an estimate of ventricular volume in those for whom there is concern for hydrocephalus. ${ }^{16}$

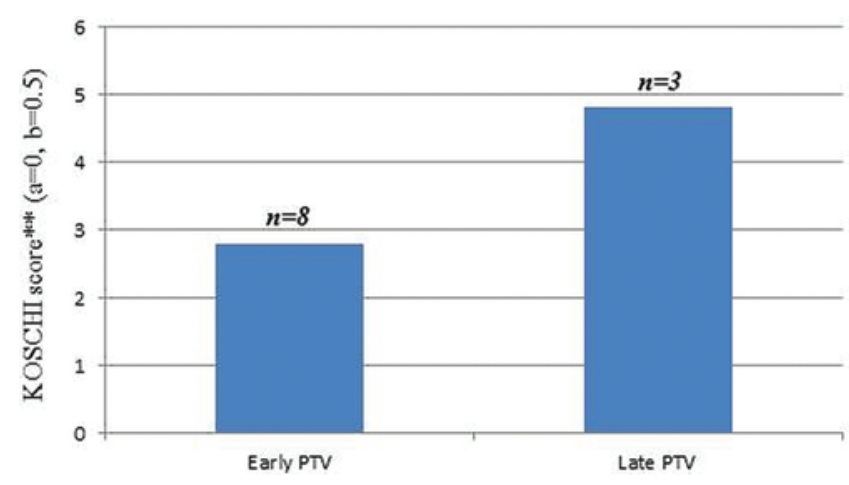

FIG. 2. Bar graph showing KOSCHI scores at the time of inpatient discharge in NAHT patients stratified by early or late PTV.

Pediatric patients with TBI can develop delayed hydrocephalus: $20 \%$ of patients with severe injuries, ${ }^{13} 29.5 \%$ after a decompressive craniectomy (accidental trauma), ${ }^{7}$ and $40 \%$ of patients with pediatric TBI (accidental and nonaccidental trauma). ${ }^{9}$ It's unclear from these studies what the natural history of developing hydrocephalus is in the NAHT population. Past studies in NAHT have evaluated mostly short-term outcomes, partly due to the high rate of infant mortality within the first 24 hours. ${ }^{5}$

We evaluated hydrocephalus association by stratifying time of arrival to medical attention, development of PTV, and time to shunt-dependent hydrocephalus. By the time of inpatient discharge, only 2 patients required a permanent shunt ( 2 of 28 patients [7\%] and 2 of 24 surviving patients [8\%]). This is not surprising when comparing this finding to observations in the reported literature that intraparenchymal hypodensities allow for more brain compliance and thus greater ventricular enlargement and blunting of cortical subarachnoid spaces as seen in our few cases involving SAH. This observation was not statistically significant, and several possible explanations may be the following: 1) poor clinical status at admission may not have allowed enough time to evaluate for development of delayed hydrocephalus, 2) a limited population was evaluated in this cohort of perpetrator-identified cases only, and 3) using dye-injection CT cisternography made direct assessment impossible. ${ }^{13}$

There has been concern that decompressive craniectomies themselves may contribute to the development of hydrocephalus. When we assessed the role of this procedure in our population, we found a statistically significant association between decompressive craniectomy and PTV. Mathematical modeling for hydrocephalus, as described in the article by Rekate et al., ${ }^{17}$ may explain why removal of part of the cranium can cause significant change in CSF dynamics. Hochwald et al. ${ }^{6}$ demonstrated blunted CSF absorption after craniectomies and investigated impaired periventricular venous circulation. In the present study, we could not address the effect of craniectomy size directly. It is possible that large craniectomies with a superior border within $2.5 \mathrm{~cm}$ of the sagittal sinus can lead to a greater incidence of PTV. ${ }^{2}$ It's unclear whether this parameter is age dependent. Importantly, selection bias in offering a decompressive craniectomy to individuals with neurological compromise likely may be brought about by signifi- 
cant intracranial findings such as hypodensities related to stroke or intracranial hemorrhage, thus having a greater propensity toward developing PTV.

\section{Conclusions}

This is the first report to identify the cumulative indices of ventriculomegaly and shunt-dependent hydrocephalus after NAHT. We observed the rate of PTV in our cohort to be similar to that after accidental TBI, and this may, in part, be related to decompressive craniectomies. Furthermore, we observed a low rate of subsequent shunt-dependent hydrocephalus. Potentially a larger collaborative prospective accrual of patients undergoing standardized hydrocephalus assessment and treatment with shunt placement protocols may confirm the observations reported here.

\section{Acknowledgments}

We thank Drs. Reginald Guerriero (Harvard University) and Aditee P. Narayan (Duke University) for critical comments and Ms. Nina Kohn and the Feinstein Institute for Medical Research for statistical assistance.

\section{References}

1. Calvert S, Miller HE, Curran A, Hameed B, McCarter R, Edwards RJ, et al: The King's Outcome Scale for Childhood Head Injury and injury severity and outcome measures in children with traumatic brain injury. Dev Med Child Neurol 50:426-431, 2008

2. De Bonis P, Pompucci A, Mangiola A, Rigante L, Anile C: Post-traumatic hydrocephalus after decompressive craniectomy: an underestimated risk factor. J Neurotrauma 27:19651970,2010

3. Dias MS, Backstrom J, Falk M, Li V: Serial radiography in the infant shaken impact syndrome. Pediatr Neurosurg 29:77-85, 1998

4. Esernio-Jenssen D, Tai J, Kodsi S: Abusive head trauma in children: a comparison of male and female perpetrators. Pediatrics 127:649-657, 2011

5. Graupman P, Winston KR: Nonaccidental head trauma as a cause of childhood death. J Neurosurg 104 (4 Suppl):245-250, 2006

6. Hochwald GM, Epstein F, Malhan C, Ransohoff J: The relationship of compensated to decompensated hydrocephalus in the cat. J Neurosurg 39:694-697, 1973

7. Jagannathan J, Okonkwo DO, Dumont AS, Ahmed H, Bahari A, Prevedello DM, et al: Outcome following decompressive craniectomy in children with severe traumatic brain injury: a 10 -year single-center experience with long-term follow up. $\mathbf{J}$ Neurosurg 106 (4 Suppl):268-275, 2007

8. Jayawant S, Rawlinson A, Gibbon F, Price J, Schulte J, Sharples P, et al: Subdural haemorrhages in infants: population based study. BMJ 317:1558-1561, 1998

9. Kan P, Amini A, Hansen K, White GL Jr, Brockmeyer DL, Walker ML, et al: Outcomes after decompressive craniectomy for severe traumatic brain injury in children. J Neurosurg 105 (5 Suppl):337-342, 2006

10. Kelly P, Farrant B: Shaken baby syndrome in New Zealand, 2000-2002. J Paediatr Child Health 44:99-107, 2008

11. Kesler H, Dias MS, Shaffer M, Rottmund C, Cappos K, Thomas NJ: Demographics of abusive head trauma in the Commonwealth of Pennsylvania. J Neurosurg Pediatr 1:351-356, 2008

12. Ludwig S, Warman M: Shaken baby syndrome: a review of 20 cases. Ann Emerg Med 13:104-107, 1984
13. Marmarou A, Foda MA, Bandoh K, Yoshihara M, Yamamoto T, Tsuji O, et al: Posttraumatic ventriculomegaly: hydrocephalus or atrophy? A new approach for diagnosis using CSF dynamics. J Neurosurg 85:1026-1035, 1996

14. Mazzini L, Campini R, Angelino E, Rognone F, Pastore I, Oliveri G: Posttraumatic hydrocephalus: a clinical, neuroradiologic, and neuropsychologic assessment of long-term outcome. Arch Phys Med Rehabil 84:1637-1641, 2003

15. Poca MA, Sahuquillo J, Mataró M, Benejam B, Arikan F, Báguena M: Ventricular enlargement after moderate or severe head injury: a frequent and neglected problem. J Neurotrauma 22:1303-1310, 2005

16. Ragan DK, Cerqua J, Nash T, McKinstry RC, Shimony JS, Jones BV, et al: The accuracy of linear indices of ventricular volume in pediatric hydrocephalus: technical note. J Neurosurg Pediatr 15:547-551, 2015

17. Rekate HL, Brodkey JA, Chizeck HJ, el Sakka W, Ko WH: Ventricular volume regulation: a mathematical model and computer simulation. Pediatr Neurosci 14:77-84, 1988

18. Starling SP, Patel S, Burke BL, Sirotnak AP, Stronks S, Rosquist P: Analysis of perpetrator admissions to inflicted traumatic brain injury in children. Arch Pediatr Adolesc Med 158:454-458, 2004

19. Stiver SI: Complications of decompressive craniectomy for traumatic brain injury. Neurosurg Focus 26(6):E7, 2009

20. Vadivelu S, Esernio-Jenssen D, Rekate HL, Narayan RK, Mittler MA, Schneider SJ: Delay in arrival to care in perpetrator-identified nonaccidental head trauma: observations and outcomes. World Neurosurg 84:1340-1346, 2015

21. Vinchon M, de Foort-Dhellemmes S, Desurmont M, Delestret I: Confessed abuse versus witnessed accidents in infants: comparison of clinical, radiological, and ophthalmological data in corroborated cases. Childs Nerv Syst 26:637-645, 2010

22. Waziri A, Fusco D, Mayer SA, McKhann GM II, Connolly ES Jr: Postoperative hydrocephalus in patients undergoing decompressive hemicraniectomy for ischemic or hemorrhagic stroke. Neurosurgery 61:489-494, 2007

\section{Disclosures}

The authors report no conflict of interest concerning the materials or methods used in this study or the findings specified in this paper.

\section{Author Contributions}

Conception and design: Vadivelu. Acquisition of data: Vadivelu, Esernio-Jenssen. Analysis and interpretation of data: Vadivelu, Rekate. Drafting the article: Vadivelu. Critically revising the article: Vadivelu, Rekate. Reviewed submitted version of manuscript: Vadivelu, Rekate, Esernio-Jenssen. Approved the final version of the manuscript on behalf of all authors: Vadivelu. Statistical analysis: Vadivelu. Administrative/technical/material support: Mittler, Schneider. Study supervision: all authors.

\section{Supplemental Information \\ Previous Presentations}

This work was presented in part at the 2011 AANS Annual Scientific Meeting, Denver, Colorado, April 9-13, 2011, and the 2011 Annual Meeting of the Congress of Neurological Surgeons, Washington, DC, October 1-6, 2011.

\section{Correspondence}

Sudhakar Vadivelu, Division of Pediatric Neurosurgery, Cincinnati Children's Hospital Medical Center, 3333 Burnett Ave., Cincinnati, OH 45040. email: sudhakar.vadivelu@cchmc.org. 